\title{
Azimuthal asymmetries of charged hadrons produced by high-energy muons scattered off longitudinally polarised deuterons
}

\author{
The COMPASS Collaboration
}

M.G. Alekseev ${ }^{28}$, V.Yu. Alexakhin ${ }^{7}$, Yu. Alexandrov ${ }^{15}$, G.D. Alexeev ${ }^{7}$, A. Amoroso ${ }^{27}$, A. Austregesilo $^{10,17}$, B. Badelek ${ }^{30}$, F. Balestra ${ }^{27}$, J. Barth ${ }^{4}$, G. Baum ${ }^{1}$, Y. Bedfer ${ }^{22}$, J. Bernhard ${ }^{13}$, R. Bertini ${ }^{27}$, M. Bettinelli ${ }^{16}$, R. Birsa ${ }^{24}$, J. Bisplinghoff ${ }^{3}$, P. Bordalo ${ }^{12, b}$, F. Bradamante ${ }^{25}$, A. Bravar ${ }^{24}$, A. Bressan ${ }^{25}$, G. Brona ${ }^{10,30}$, E. Burtin ${ }^{22}$, M.P. Bussa ${ }^{27}$, D. Chaberny $^{13}$, M. Chiosso ${ }^{27}$, S.U. Chung ${ }^{17}$, A. Cicuttin ${ }^{26}$, M. Colantoni ${ }^{28}$, M.L. Crespo $^{26}$, S. Dalla Torre ${ }^{24}$, S. Das ${ }^{6}$, S.S. Dasgupta ${ }^{6}$, O.Yu. Denisov ${ }^{10,28}$, L. Dhara ${ }^{6}$, V. Diaz ${ }^{26}$, S.V. Donskov ${ }^{21}$, N. Doshita $^{2,32}$, V. Duic ${ }^{25}$, W. Dünnweber ${ }^{16}$, A. Efremov ${ }^{7}$, A. El Alaoui ${ }^{22}$, P.D. Eversheim ${ }^{3}$, W. Eyrich ${ }^{8}$, M. Faessler ${ }^{16}$, A. Ferrero Fe $^{22}$, A. Filin ${ }^{21}$, M. Finger ${ }^{19}$, M. Finger Jr. ${ }^{7}$, H. Fischer ${ }^{9}$, C. Franco ${ }^{12}$, J.M. Friedrich ${ }^{17}$, R. Garfagnini ${ }^{27}$, F. Gautheron $^{2}$, O.P. Gavrichtchouk ${ }^{7}$, R. Gazda ${ }^{30}$, S. Gerassimov ${ }^{15,17}$, R. Geyer ${ }^{16}$, M. Giorgi ${ }^{25}$, I. Gnesi ${ }^{27}$, B. Gobbo ${ }^{24}$, S. Goertz ${ }^{2,4}$, S. Grabmüller ${ }^{17}$, A. Grasso ${ }^{27}$, B. Grube ${ }^{17}$, R. Gushterski ${ }^{7}$, A. Guskov ${ }^{7}$, F. Haas ${ }^{17}$, D. von Harrach ${ }^{13}$, T. Hasegawa ${ }^{14}$, F.H. Heinsius ${ }^{9}$, F. Herrmann ${ }^{9}$, C. Heß ${ }^{2}$, F. Hinterberger ${ }^{3}$, N. Horikawa ${ }^{18, c}$, Ch. Höppner ${ }^{17}$, N. d'Hose $^{22}$, C. Ilgner ${ }^{10,16}$, S. Ishimoto ${ }^{18, d}$, O. Ivanov ${ }^{7}$, Yu. Ivanshin ${ }^{7}$, T. Iwata ${ }^{32}$, R. Jahn ${ }^{3}$, P. Jasinski ${ }^{13}$, G. Jegou ${ }^{22}$, R. Joosten $^{3}$, E. Kabuß ${ }^{13}$, D. Kang ${ }^{9}$, B. Ketzer ${ }^{17}$, G.V. Khaustov ${ }^{21}$, Yu.A. Khokhlov ${ }^{21}$, Yu. Kisselev ${ }^{2}$, F. Klein ${ }^{4}$, K. Klimaszewski ${ }^{30}$, S. Koblitz ${ }^{13}$, J.H. Koivuniemi ${ }^{2}$, V.N. Kolosov ${ }^{21}$, K. Kondo ${ }^{2,32}$, K. Königsmann ${ }^{9}$, R. Konopka ${ }^{17}$, I. Konorov ${ }^{15,17}$, V.F. Konstantinov ${ }^{21}$, A. Korzenev ${ }^{13, \text { e }}$, A.M. Kotzinian ${ }^{27}$, O. Kouznetsov ${ }^{7,22}$, K. Kowalik ${ }^{30,22}$, M. Krämer ${ }^{17}$, A. Kral ${ }^{20}$, Z.V. Kroumchtein ${ }^{7}$, R. Kuhn ${ }^{17}$, F. Kunne ${ }^{22}$, K. Kurek ${ }^{30}$, L. Lauser ${ }^{9}$, J.M. Le Goff ${ }^{22}$, A.A. Lednev ${ }^{21}$, A. Lehmann ${ }^{8}$, S. Levorato ${ }^{25}$, J. Lichtenstadt ${ }^{23}$, T. Liska ${ }^{20}$, A. Maggiora ${ }^{28}$, M. Maggiora ${ }^{27}$, A. Magnon ${ }^{22}$,

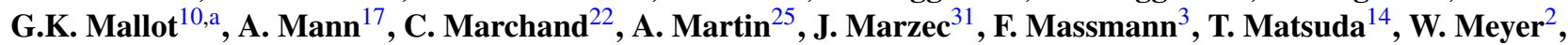
T. Michigami ${ }^{32}$, Yu.V. Mikhailov ${ }^{21}$, M.A. Moinester ${ }^{23}$, A. Mutter ${ }^{9,13}$, A. Nagaytsev ${ }^{7}$, T. Nagel ${ }^{17}$, J. Nassalski ${ }^{30}$, $^{\dagger}$, T. Negrini $^{3}$, F. Nerling ${ }^{9}$, S. Neubert ${ }^{17}$, D. Neyret $^{22}$, V.I. Nikolaenko ${ }^{21}$, A.S. Nunes ${ }^{12}$, A.G. Olshevsky ${ }^{7}$, M. Ostrick ${ }^{13}$, A. Padee $^{31}$, R. Panknin ${ }^{4}$, D. Panzieri ${ }^{29}$, B. Parsamyan ${ }^{27}$, S. Paul ${ }^{17}$, B. Pawlukiewicz-Kaminska ${ }^{30}$, E. Perevalova ${ }^{7}$, G. Pesaro $^{25}$, D.V. Peshekhonov ${ }^{7}$, G. Piragino ${ }^{27}$, S. Platchkov ${ }^{22}$, J. Pochodzalla ${ }^{13}$, J. Polak ${ }^{11,25}$, V.A. Polyakov ${ }^{21}$, G. Pontecorvo ${ }^{7}$, J. Pretz ${ }^{4}$, C. Quintans ${ }^{12}$, J.-F. Rajotte ${ }^{16}$, S. Ramos $^{12, b}$, V. Rapatsky ${ }^{7}$, G. Reicherz ${ }^{2}$, A. Richter ${ }^{8}$, F. Robinet $^{22}$, E. Rocco $^{27}$, E. Rondio ${ }^{30}$, D.I. Ryabchikov ${ }^{21}$, V.D. Samoylenko ${ }^{21}$, A. Sandacz ${ }^{30}$ H. Santos $^{12}$, M.G. Sapozhnikov ${ }^{7}$, S. Sarkar ${ }^{6}$, I.A. Savin ${ }^{7}$, G. Sbrizzai ${ }^{25}$, P. Schiavon ${ }^{25}$, C. Schill ${ }^{9}$, T. Schlüter ${ }^{16}$, L. Schmitt ${ }^{17, f}$, S. Schopferer ${ }^{9}$, W. Schröder ${ }^{8}$, O.Yu. Shevchenko ${ }^{7}$, H.-W. Siebert ${ }^{13}$, L. Silva ${ }^{12}$, L. Sinha ${ }^{6}$, A.N. Sissakian ${ }^{7, \dagger}$, M. Slunecka ${ }^{7}$, G.I. Smirnov ${ }^{7}$, S. Sosio ${ }^{27}$, F. Sozzi ${ }^{25}$, A. Srnka ${ }^{5}$, M. Stolarski ${ }^{10}$, M. Sulc ${ }^{11}$, R. Sulej ${ }^{31}$, S. Takekawa ${ }^{25}$, S. Tessaro $^{24}$, F. Tessarotto ${ }^{24}$, A. Teufel ${ }^{8}$, L.G. Tkatchev ${ }^{7}$, S. Uhl ${ }^{17}$, I. Uman ${ }^{16}$, M. Virius ${ }^{20}$, N.V. Vlassov ${ }^{7}$, A. Vossen ${ }^{9}$, Q. Weitzel $^{17}$, R. Windmolders ${ }^{4}$, W. Wiślicki ${ }^{30}$, H. Wollny ${ }^{9}$, K. Zaremba ${ }^{31}$, M. Zavertyaev ${ }^{15}$, E. Zemlyanichkina ${ }^{7}$, M. Ziembicki ${ }^{31}$, J. Zhao ${ }^{13,24}$, N. Zhuravlev ${ }^{7}$, A. Zvyagin ${ }^{16}$

\footnotetext{
${ }^{1}$ Universität Bielefeld, Fakultät für Physik, 33501 Bielefeld, Germany ${ }^{\mathrm{g}}$

${ }^{2}$ Universität Bochum, Institut für Experimentalphysik, 44780 Bochum, Germanyg

${ }^{3}$ Universität Bonn, Helmholtz-Institut für Strahlen- und Kernphysik, 53115 Bonn, Germany ${ }^{g}$

${ }^{4}$ Universität Bonn, Physikalisches Institut, 53115 Bonn, Germany ${ }^{\mathrm{g}}$

${ }^{5}$ Institute of Scientific Instruments, AS CR, 61264 Brno, Czech Republic ${ }^{\mathrm{h}}$

${ }^{6}$ Matrivani Institute of Experimental Research \& Education, Calcutta 700 030, India ${ }^{\mathrm{i}}$

${ }^{7}$ Joint Institute for Nuclear Research, 141980 Dubna, Moscow region, Russia ${ }^{j}$

${ }^{8}$ Universität Erlangen-Nürnberg, Physikalisches Institut, 91054 Erlangen, Germany ${ }^{\mathrm{g}}$

${ }^{9}$ Universität Freiburg, Physikalisches Institut, 79104 Freiburg, Germany ${ }^{\mathrm{g}}$

${ }^{10}$ CERN, 1211 Geneva 23, Switzerland

${ }^{11}$ Technical University in Liberec, 46117 Liberec, Czech Republic ${ }^{h}$

${ }^{12}$ LIP, 1000-149 Lisbon, Portugal ${ }^{\mathrm{k}}$

${ }^{13}$ Universität Mainz, Institut für Kernphysik, 55099 Mainz, Germany ${ }^{\mathrm{g}}$

${ }^{14}$ University of Miyazaki, Miyazaki 889-2192, Japan ${ }^{1}$

${ }^{15}$ Lebedev Physical Institute, 119991 Moscow, Russia
} 
${ }^{16}$ Ludwig-Maximilians-Universität München, Department für Physik, 80799 Munich, Germany ${ }^{\mathrm{g}, \mathrm{m}}$

${ }^{17}$ Technische Universität München, Physik Department, 85748 Garching, Germanyg,m

${ }^{18}$ Nagoya University, 464 Nagoya, Japan ${ }^{1}$

${ }^{19}$ Charles University in Prague, Faculty of Mathematics and Physics, 18000 Prague, Czech Republic ${ }^{\text {h }}$

${ }^{20}$ Czech Technical University in Prague, 16636 Prague, Czech Republic ${ }^{\mathrm{h}}$

${ }^{21}$ State Research Center of the Russian Federation, Institute for High Energy Physics, 142281 Protvino, Russia

${ }^{22}$ CEA IRFU/SPhN Saclay, 91191 Gif-sur-Yvette, France

${ }^{23}$ Tel Aviv University, School of Physics and Astronomy, 69978 Tel Aviv, Israel ${ }^{\mathrm{n}}$

${ }^{24}$ Trieste Section of INFN, 34127 Trieste, Italy

${ }^{25}$ University of Trieste, Department of Physics and Trieste Section of INFN, 34127 Trieste, Italy

${ }^{26}$ Abdus Salam ICTP and Trieste Section of INFN, 34127 Trieste, Italy

${ }^{27}$ University of Turin, Department of Physics and Torino Section of INFN, 10125 Turin, Italy

${ }^{28}$ Torino Section of INFN, 10125 Turin, Italy

${ }^{29}$ University of Eastern Piedmont, 1500 Alessandria, and Torino Section of INFN, 10125 Turin, Italy

${ }^{30}$ Sołtan Institute for Nuclear Studies and University of Warsaw, 00-681 Warsaw, Poland ${ }^{\mathrm{o}}$

${ }^{31}$ Warsaw University of Technology, Institute of Radioelectronics, 00-665 Warsaw, Poland ${ }^{\circ}$

${ }^{32}$ Yamagata University, Yamagata, 992-8510, Japan ${ }^{1}$

Received: 9 July 2010 / Revised: 14 September 2010 / Published online: 15 October 2010

(C) The Author(s) 2010. This article is published with open access at Springerlink.com

\begin{abstract}
Azimuthal asymmetries in semi-inclusive production of positive $\left(h^{+}\right)$and negative hadrons $\left(h^{-}\right)$have been measured by scattering $160 \mathrm{GeV}$ muons off longitudinally polarised deuterons at CERN. The asymmetries were decomposed in several terms according to their expected modulation in the azimuthal angle $\phi$ of the outgoing hadron. Each term receives contributions from one or several spin and transverse-momentum-dependent parton distribution and fragmentation functions. The amplitudes of all $\phi$-modulation terms of the hadron asymmetries integrated
\end{abstract}

a e-mail: Gerhard.Mallot@cern.ch

${ }^{\mathrm{b}}$ Also at IST, Universidade Técnica de Lisboa, Lisbon, Portugal.

${ }^{\mathrm{c}}$ Also at Chubu University, Kasugai, Aichi, 487-8501 Japan ${ }^{1}$.

${ }^{\mathrm{d}}$ Also at KEK, 1-1 Oho, Tsukuba, Ibaraki, 305-0801 Japan.

${ }^{\mathrm{e} O n}$ leave of absence from JINR Dubna.

${ }^{\mathrm{f}}$ Also at GSI mbH, Planckstr. 1, D-64291 Darmstadt, Germany.

${ }^{\mathrm{g}}$ Supported by the German Bundesministerium für Bildung und Forschung.

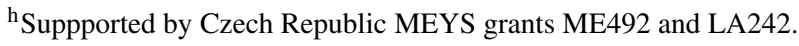

${ }^{i}$ Supported by SAIL (CSR), Govt. of India.

${ }^{\mathrm{j}}$ Supported by CERN-RFBR grants 08-02-91009 and 08-02-91013.

${ }^{\mathrm{k}}$ Supported by the Portuguese FCT-Fundação para a Ciência e Tecnologia grants POCTI/FNU/49501/2002 and POCTI/FNU/50192/2003.

${ }^{1}$ Supported by the MEXT and the JSPS under the Grants No. 18002006, No. 20540299 and No. 18540281; Daiko Foundation and Yamada Foundation.

${ }^{\mathrm{m}}$ Supported by the DFG cluster of excellence 'Origin and Structure of the Universe' (www.universe-cluster.de).

${ }^{\mathrm{n}}$ Supported by the Israel Science Foundation, founded by the Israel Academy of Sciences and Humanities.

${ }^{\circ}$ Supported by Ministry of Science and Higher Education grant $41 / \mathrm{N}$ CERN/2007/0.

$\dagger$ Deceased. over the kinematic variables are found to be consistent with zero within statistical errors, while the constant terms are nonzero and equal for $h^{+}$and $h^{-}$within the statistical errors. The dependencies of the $\phi$-modulated terms versus the Bjorken momentum fraction $x$, the hadron fractional momentum $z$, and the hadron transverse momentum $p_{h}^{T}$ were studied. The $x$ dependence of the constant terms for both positive and negative hadrons is in agreement with the longitudinal double-spin hadron asymmetries, measured in semiinclusive deep-inelastic scattering. The $x$ dependence of the $\sin \phi$-modulation term is less pronounced than that in the corresponding HERMES data. All other dependencies of the $\phi$-modulation amplitudes are consistent with zero within the statistical errors.

\section{Introduction}

Starting from the first polarised lepton scattering experiments at SLAC $[1,2]$ and at CERN by the EMC $[3,4]$, the longitudinal spin structure of the nucleon has been investigated over the past 20 years by the SMC [5], HERMES [6, 7], CLAS [8-10] and COMPASS [11-15] Collaborations. The cross-section asymmetries $A_{1}$ and $A_{1}^{h}$ were measured respectively in inclusive Deep Inelastic Scattering (DIS)

$\vec{\ell}+\vec{N} \rightarrow \ell^{\prime}+X$

and Semi-Inclusive Deep-Inelastic Scattering (SIDIS)

$\vec{\ell}+\vec{N} \rightarrow \ell^{\prime}+h+X$

of longitudinally polarised leptons $(\vec{\ell})$ off longitudinally polarised nucleons $(\vec{N})$. SIDIS, where in addition to the scat- 
Fig. 1 (a) The kinematics of the SIDIS process shown for antiparallel target polarisation $P_{\|}$w.r.t. the beam momentum $\left(\phi_{S}=\pi\right)$. (b) Squared modulus of the matrix element of the SIDIS reaction

$\ell+\vec{N} \rightarrow \ell^{\prime}+h+X$ summed over the final states $X$

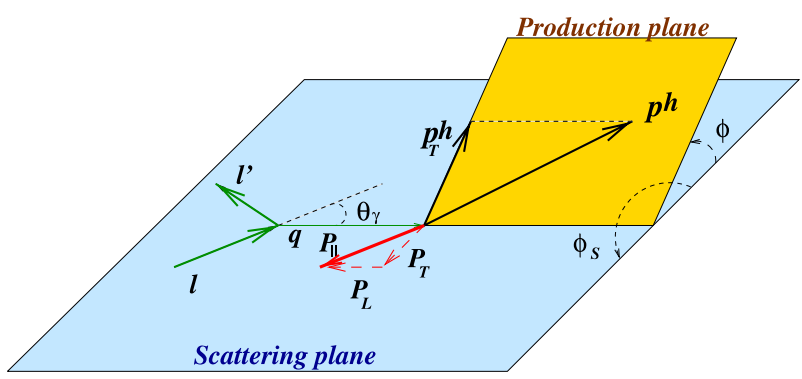

(a)

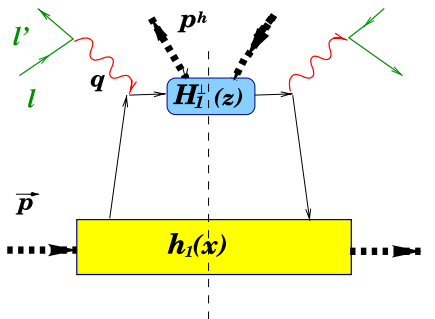

(b) tered lepton a hadron $h$ is detected, gives access to the individual quark spin distributions. From the measured spindependent asymmetries the contributions of quark spins to the spin of nucleons as well as the spin quark distribution functions for valence and sea quarks have been determined.

The hadron transverse momentum leads to a dependence of the SIDIS cross-section on the hadron azimuthal angle $\phi$ (Fig. 1a) and to asymmetries in the hadron production. The asymmetries have been predicted [16-18] and elaborated on in a number of theoretical papers (see Refs. [1924] and references therein). The azimuthal asymmetries are related to transverse-momentum-dependent Parton Distribution Functions (PDF) and polarised and nonpolarised Parton Fragmentation Functions (PFF). They can depend on the transverse or longitudinal component of the quark spin. These asymmetries were first observed by SMC [25], HERMES [26, 27] and CLAS [28, 29]. Further studies were performed both with transversely polarised targets by HERMES (proton) [30-32] and COMPASS (deuteron, proton) [33-36] and with longitudinally polarised targets by HERMES (proton, deuteron) [37, 38] and CLAS (proton) [39]. Some of the measured azimuthal asymmetries (e.g. the socalled "Collins asymmetry") for the proton are rather large, reaching up to $10 \%$ [30-32], others however (e.g. the socalled "pretzelosity"), do not exceed a couple of percent [40] and their investigation requires very high statistics. The asymmetries for the deuteron are found to be much smaller than those for the proton indicating opposite signs of contributions from $u$ and $d$ quarks [22, 23].

A search for azimuthal asymmetries for unidentified hadrons with longitudinally polarised deuterons is presented below. These new data will test in a wide $\left(x, Q^{2}\right)$ range the existence of still unobserved azimuthal asymmetries connected to several transverse-momentum-dependent PDFs.

The Paper is organised as follows. In Sect. 2 a short theoretical overview with the basic formulae is given. The analysis method and the data selection are described in Sects. 3 and 4, respectively. The results are presented in Sect. 5 and their stability and systematic uncertainty are discussed in Sect. 6, followed by the conclusions in Sect. 7 .

\section{Theoretical framework}

The kinematics of the SIDIS reaction is shown in Fig. 1a. The 4-momenta of the incident and scattered muon are denoted by $\ell$ and $\ell^{\prime}$, respectively. The momentum transfer is given by $q=\ell-\ell^{\prime}$ with $Q^{2}=-q^{2}$ and $\theta_{\gamma}$ is the angle of the momentum $\vec{q}$ of the virtual photon with respect to the beam. The vectors $p^{h}$ and $P_{\|}$are the hadron momentum and the longitudinal target polarisation, respectively. Their transverse components $p_{T}^{h}$ and $P_{T}$ are defined with respect to the virtual photon momentum. The longitudinal component $\left|P_{L}\right|=P_{\|} \cos \theta_{\gamma}$ is approximately equal to $P_{\|}$due to the smallness of the angle $\theta_{\gamma}$. The small transverse component is equal to $\left|P_{T}\right|=P_{\|} \sin \left(\theta_{\gamma}\right)$ where $\sin \left(\theta_{\gamma}\right) \approx 2(M x / Q) \sqrt{1-y}, M$ is the nucleon mass and $y=q \cdot p / p \cdot \ell$ is the fraction of the muon energy lost in the laboratory reference frame. The angle $\phi$ is the azimuthal angle between the scattering plane and the hadron production plane and $\phi_{S}$ is the angle of the target polarisation vector with respect to the lepton scattering plane. The invariant mass squared of the virtual photon-nucleon system $W^{2}$, the Bjorken variable $x$ and the hadron momentum fraction $z$, characterising SIDIS together with $Q^{2}$ and $y$, are defined as $W^{2}=(p+q)^{2}, x=Q^{2} / 2 p \cdot q, z=p \cdot p^{h} / p \cdot q$ where $p$ is the 4-momentum of the incident nucleon.

The general expression of the total differential crosssection for the SIDIS reaction is a linear function of the muon beam polarisation $P_{\mu}$ and of the target polarisation components $P_{L}$ and $P_{T}$

$$
\begin{aligned}
\mathrm{d} \sigma= & \mathrm{d} \sigma_{00}+P_{\mu} \mathrm{d} \sigma_{L 0}+P_{L}\left(\mathrm{~d} \sigma_{0 L}+P_{\mu} \mathrm{d} \sigma_{L L}\right) \\
& +\left|P_{T}\right|\left(\mathrm{d} \sigma_{0 T}+P_{\mu} \mathrm{d} \sigma_{L T}\right)
\end{aligned}
$$

where the first (second) subscript of the partial crosssections refers to the beam (target) polarisation.

The asymmetry $a(\phi)$ for hadron production from a longitudinally polarised target is defined by

$$
\begin{aligned}
a(\phi) & =\frac{\mathrm{d} \sigma^{\leftarrow \Rightarrow}-\mathrm{d} \sigma^{\leftarrow \Leftarrow}}{\left|P_{L}\right|\left(\mathrm{d} \sigma^{\leftarrow \Rightarrow}+\mathrm{d} \sigma^{\leftarrow \Leftarrow)}\right)} \\
& =-\frac{\mathrm{d} \sigma_{0 L}+P_{\mu} \mathrm{d} \sigma_{L L}-\tan \theta_{\gamma}\left(\mathrm{d} \sigma_{0 T}+P_{\mu} \mathrm{d} \sigma_{L T}\right)}{\mathrm{d} \sigma_{00}+P_{\mu} \mathrm{d} \sigma_{L 0}},
\end{aligned}
$$


where $\Rightarrow$ or $\Leftarrow$ denotes the target polarisation along or opposite to the muon beam direction and $\leftarrow$ denotes the beam polarisation, which is always opposite to the beam direction. The partial cross-sections $\mathrm{d} \sigma_{00}$ and $\mathrm{d} \sigma_{L 0}$ do not contribute to the numerator of the asymmetry (see (4)) while $\mathrm{d} \sigma_{0 T}$ and $\mathrm{d} \sigma_{L T}$ are suppressed by the small value of $\left|P_{T}\right| /\left|P_{L}\right|=$ $\tan \theta_{\gamma} \approx 2(M x / Q) \sqrt{1-y}$.

In the parton model (one-photon approximation, handbagtype diagram) the squared modulus of the matrix element of the SIDIS reaction is represented by a diagram of the type shown in Fig. 1b. The chiral-odd transversity ${ }^{1}$ PDF $h_{1}(x)$ coupled to the chiral-odd Collins PFF $H_{1}^{\perp}(z)$ is given as an example. Each of the partial cross-sections in (3) is characterised by several terms including a convolution of PDF and PFF multiplied by a function of the azimuthal angle of the outgoing hadron. Ignoring the pure twist-3 ("tilde") fragmentation functions and retaining only terms up to order $(M / Q)$, the contributions to (4) for unpolarised or spin-zero hadron production have the forms

$$
\begin{aligned}
\mathrm{d} \sigma_{00} \propto & x f_{1}(x) \otimes D_{1}(z)+\epsilon x h_{1}^{\perp}(x) \otimes H_{1}^{\perp}(z) \cos (2 \phi) \\
& +\sqrt{2 \epsilon(1+\epsilon)} \frac{M}{Q} x^{2}\left(h(x) \otimes H_{1}^{\perp}(z)\right. \\
& \left.+f^{\perp}(x) \otimes D_{1}(z)\right) \cos \phi \\
\mathrm{d} \sigma_{L 0} \propto & \sqrt{2 \epsilon(1-\epsilon)} \frac{M}{Q} x^{2}\left(e(x) \otimes H_{1}^{\perp}(z)\right. \\
& \left.+g^{\perp}(x) \otimes D_{1}^{\perp}(z)\right) \sin \phi, \\
\mathrm{d} \sigma_{0 L} \propto & \epsilon h_{1 L}^{\perp}(x) \otimes H_{1}^{\perp}(z) \sin (2 \phi) \\
& +\sqrt{2 \epsilon(1+\epsilon)} \frac{M}{Q} x^{2}\left(h_{L}(x) \otimes H_{1}^{\perp}(z)\right. \\
& \left.+f_{L}^{\perp}(x) \otimes D_{1}(z)\right) \sin \phi \\
\mathrm{d} \sigma_{L L} \propto & \sqrt{1-\epsilon^{2}} x g_{1 L}(x) \otimes D_{1}(z) \\
& +\sqrt{2 \epsilon(1-\epsilon)} \frac{M}{Q} x^{2}\left(g_{L}^{\perp}(x) \otimes D_{1}(z)\right. \\
& \left.+e_{L}(x) \otimes H_{1}^{\perp}(z)\right) \cos \phi \\
\mathrm{d} \sigma_{0 T} \propto & \epsilon\left\{x h_{1}(x) \otimes H_{1}^{\perp}(z) \sin \left(\phi+\phi_{S}\right)\right. \\
& \left.+x h_{1 T}^{\perp}(x) \otimes H_{1}^{\perp}(z) \sin \left(3 \phi-\phi_{S}\right)\right\} \\
& +x f_{1 T}^{\perp}(x) \otimes D_{1}(z) \sin \left(\phi-\phi_{S}\right), \\
\mathrm{d} \sigma_{L T} \propto & \sqrt{1-\epsilon^{2}} x g_{1 T}(x) \otimes D_{1}(z) \cos \left(\phi-\phi_{S}\right),
\end{aligned}
$$

where the target spin angle is $\phi_{S}=0$ or $\pi$. The ratio of the virtual-photon flux with longitudinal to that with transverse polarisation is given by $\epsilon \approx 2(1-y) /\left(2-2 y+y^{2}\right)$.

${ }^{1}$ In this Paper we follow the Amsterdam notations [20, 24] for PDF and PFF.
The symbol $\otimes$ represents a convolution of a PDF and a PFF weighted by a function of transverse momenta as defined in (4.1)-(4.19) of Ref. [24] where also the exact expressions of the cross-sections are given.

Some of the asymmetry modulations arising from (4) and (5) have already been observed in experiments with either transversely or longitudinally polarised targets. These asymmetries involve the $\sin \phi$ modulation in $\mathrm{d} \sigma_{0 L}$ due to the twist-3 PDFs $h_{L}$ and $f_{L}^{\perp}$ and that in $\mathrm{d} \sigma_{0 T}$ due to transversity $h_{1}$ and the Sivers PDF $f_{1 T}^{\perp}$. The $\sin (2 \phi)$ modulation in $\mathrm{d} \sigma_{0 L}$ arising from the "worm-gear" PDF $h_{1 L}^{\perp}$ has been seen also. Other asymmetries have not yet been observed experimentally. These include the $\sin (3 \phi)$ modulation in $\mathrm{d} \sigma_{0 T}$ related to the "pretzelosity" PDF and the $\cos \phi$ modulations both in $\mathrm{d} \sigma_{L T}$ due the "worm-gear" PDF $g_{1 T}$ and in $\mathrm{d} \sigma_{L L}$ due to the twist $3 g_{L}^{\perp}$ and $e_{L}$.

The aim of this study is to evaluate the azimuthal asymmetries in hadron production from the longitudinally polarised target as a manifestation of the quark-spin and transverse-momentum dependent PDFs and PFF mentioned above and to investigate the $x, z$ and $p_{T}^{h}$ dependence of the corresponding modulation amplitudes.

\section{Analysis}

The experiment was performed in the muon beam M2 at CERN with positive $160 \mathrm{GeV}$ muons. The beam is naturally polarised opposite to the muon momentum with an average polarisation $P_{\mu}=-80 \%$.

Briefly, the COMPASS setup [41] is a two-stage forward spectrometer with the world's largest polarised target, tracking detectors and particle identification detectors behind each of the two spectrometer magnets. Various tracking detectors (MICROMEGAS, GEM, Straw, MWPC, DC) provide a precise determination of the particle coordinates, while electromagnetic and hadron calorimeters, muon detectors, and a RICH provide identification of secondary electrons, muons and hadrons. The fast trigger and data acquisition systems provide for high statistics measurements.

The method of analysis takes advantage of the polarised target arrangement optimised for asymmetry measurements. In 2002-2004 the target consisted of an upstream cell ("U") and a downstream cell ("D") placed along the axis of a $2.5 \mathrm{~T}$ solenoidal magnet centred along the beam direction. The target material ${ }^{6} \mathrm{LiD}$ was kept at a low temperature in a ${ }^{3} \mathrm{He}-{ }^{4} \mathrm{He}$ dilution refrigerator. The material in the cells was polarised longitudinally with opposite orientations by Dynamic Nuclear Polarisation. The beam traverses both cells and data are taken simultaneously for both polarisations. To minimise remaining systematic effects caused by possible time-dependent variations of the acceptance, the polarisation of the cells is reversed three times per day by adiabati- 
cally inverting the solenoid field. To avoid possible systematic acceptance effects resulting from the different solenoid field orientations, after a few weeks the same polarisation configuration is realised with inverted magnetic field.

For the azimuthal-asymmetry studies double ratios $R_{f}$ of event numbers are used

$$
R_{f}(\phi)=\left[N_{+, f}^{U}(\phi) / N_{-, f}^{D}(\phi)\right] \times\left[N_{+, f}^{D}(\phi) / N_{-, f}^{U}(\phi)\right],
$$

where $N_{p, f}^{t}(\phi)$ is the number of events in a given $\phi$ bin originating from the target cell $t(t=U, D)$ with the polarisation orientation $p(p=+,-)$ and the solenoid field orientation $f(f=+,-)$ w.r.t. to the beam direction.

Using (3), (5) the number of events can be expressed as

$$
\begin{aligned}
N_{p, f}^{t}= & C_{f}^{t}(\phi) L_{p, f}^{t}\left[\left(B_{0}+B_{1} \cos \phi+B_{2} \cos 2 \phi\right.\right. \\
& \left.+B_{3} \sin \phi+\cdots\right) \\
& \left. \pm P_{p, f}^{t}\left(A_{0}+A_{1} \sin \phi+A_{2} \sin (2 \phi)+\cdots\right)\right],
\end{aligned}
$$

where $C_{f}^{t}(\phi)$ is the acceptance factor, $L_{p, f}^{t}$ is the luminosity, and $P_{p, f}^{t}$ is the absolute value of the averaged product of the measured positive or negative target polarisation and the dilution factor ${ }^{2}$ calculated for the cell $t$ [11-14]. The coefficients $B_{0}, B_{1}, \ldots$ and $A_{0}, A_{1}, \ldots$ characterise the target-spin-independent and the target-spin-dependent parts of partial cross-sections contributing to the denominator and numerator in (4), respectively.

Substituting (7) into (6), one can see that the multiplicative acceptance factors cancel out as well as the luminosity factors if the beam muons cross both cells. The ratios $R_{f}(\phi)$ thus depend only on the asymmetry $a(\phi)$ (see (4)), which can be expressed (to first order) by

$$
a_{f}(\phi)=\left[R_{f}(\phi)-1\right] /\left(P_{+, f}^{U}+P_{+, f}^{D}+P_{-, f}^{U}+P_{-, f}^{D}\right) .
$$

Since the asymmetry should not depend on the orientation of the solenoid field, one can expect $a_{+}=a_{-}$. From the data a small nonzero difference between $a_{+}$and $a_{-}$was found. This difference may be due to nonfactorisable solenoidfield-dependent contributions in (7). However, these contributions have different signs, as it was checked by Monte Carlo simulations, and cancel out in the weighted sum $a(\phi)=a_{+}(\phi) \oplus a_{-}(\phi)$. Therefore, this sum-calculated separately for each year of data taking and averaged at the end-is used for the final results.

\footnotetext{
${ }^{2}$ The dilution factor is given by the ratio of the absorption crosssections on the deuteron to that of all nuclei entering the target cells. It includes a correction for the relative polarisation of deuterons bound in ${ }^{6} \mathrm{Li}$ with respect to free deuterons. It also includes the dilution due to radiative events on the deuteron, which is taken into account by the ratio of the one-photon exchange cross-section to the total cross-section.
}

\section{Data selection}

The data selection starts from the full data set of SIDIS events taken in 2002-2004 with $Q^{2}>1(\mathrm{GeV} / c)^{2}$ and $y>$ 0.1 . For each event a reconstructed vertex with incoming and outgoing muons and one or more additional outgoing tracks is required. Applying cuts on the quality of reconstructed tracks and vertices, the target volume, the momentum of the incoming muon $(140<|\boldsymbol{l}|<180 \mathrm{GeV} / c)$, the fraction of the muon energy loss $(y<0.9)$ and the invariant mass of the virtual photon-nucleon system $\left(5<W<18 \mathrm{GeV} / c^{2}\right)$, about $96 \times 10^{6}$ of the SIDIS events remain for further analysis.

The tracks originating from SIDIS events have been identified as hadrons using the information from the hadron calorimeters HCAL1 and HCAL2 [41]. A track is considered a hadron track if it hits one of the calorimeters, the calorimeter has an associated cluster with energy greater then $5 \mathrm{GeV}$ in HCAL1 or greater then $7 \mathrm{GeV}$ in HCAL2, the coordinates of the cluster are compatible with those of the track, and the energy of the cluster is compatible with the momentum of the track. The total number of the hadrons is about $53 \times 10^{6}$.

The asymmetries are evaluated in the restricted kinematic region of $x=0.004-0.7, z=0.2-0.9, p_{T}^{h}=0.1-$ $1 \mathrm{GeV} / c$. The lower $x$-cut value corresponds to a cut on $Q^{2}>1(\mathrm{GeV} / c)^{2}$, while the highest $x$ value is determined by the acceptance. The restriction of the energy fraction of hadrons to $z>0.2$ corresponds to the c.m. Feynman variable $x_{F} \approx z-\left(p_{T}^{h^{2}}+m_{h}^{2}\right) /\left(z W^{2}\right)>0$ and assures that the hadron comes from the current fragmentation region. This cut removes almost half of the hadron tracks. The high- $z$ cut is applied to limit the influence of exclusive channels. The low- $p_{T}^{h}$ cut guarantees a good determination of the hadron angles while the high- $p_{T}^{h}$ cut corresponds kinematically to the $x_{F}$ and $W$ cuts. Distributions of the SIDIS events, from which the hadrons are selected for asymmetry evaluations, are shown in Fig. 2 vs. $Q^{2}$ and $y$. The average value of $Q^{2}$ is $3.26(\mathrm{GeV} / c)^{2}$. The distributions of the hadrons vs. $z$ and $p_{T}^{h}$ are shown in Fig. 3.

\section{Results}

The weighted sums of the azimuthal asymmetry $a(\phi)=$ $a_{+}(\phi) \oplus a_{-}(\phi)$ for negative and positive hadrons have been fitted by the function

$$
\begin{aligned}
a(\phi)= & a^{\text {const }}+a^{\sin \phi} \sin \phi+a^{\sin 2 \phi} \sin (2 \phi) \\
& +a^{\sin 3 \phi} \sin (3 \phi)+a^{\cos \phi} \cos \phi .
\end{aligned}
$$

The fit parameters $a^{f(\phi)}$ are connected to particular PDFs and PFFs in (5) and can depend on $x, z$ and $p_{T}^{h}$. First, the asymmetries $a(\phi)$ integrated over these kinematic variables 

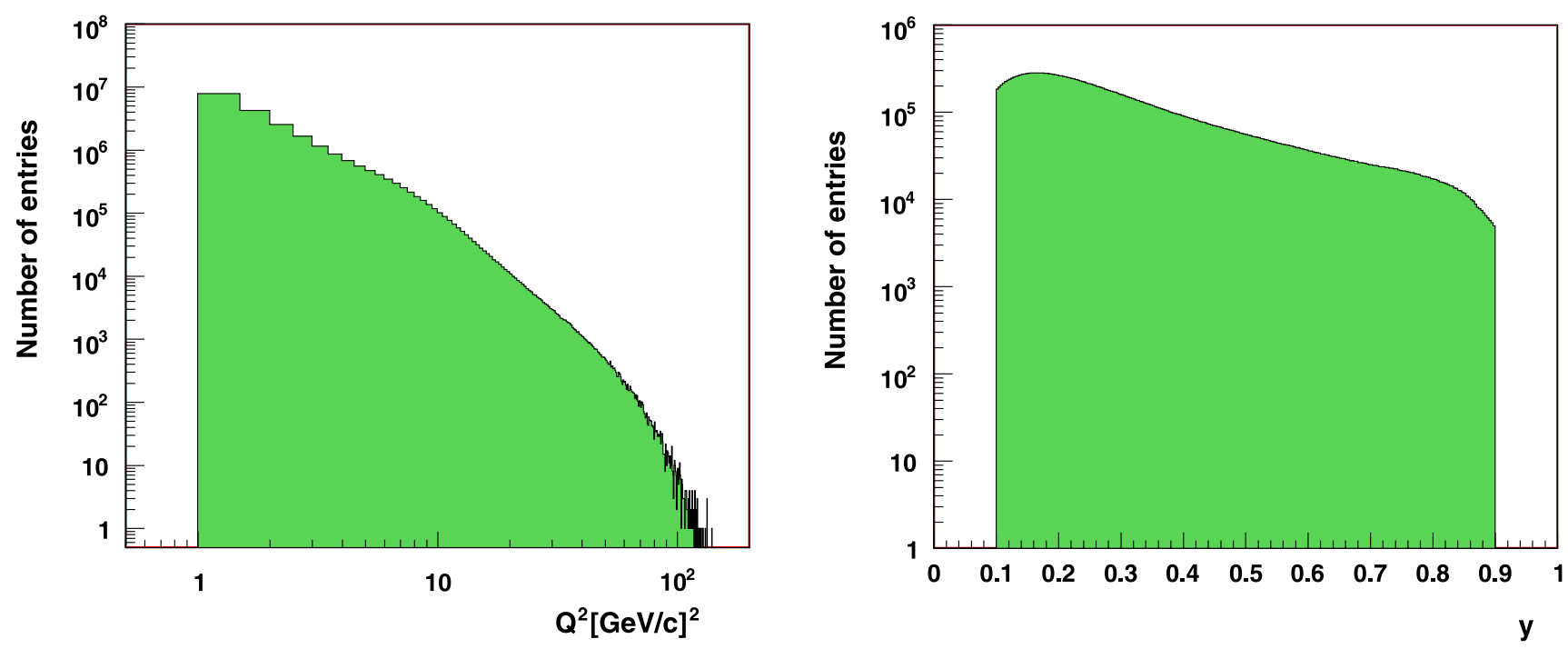

Fig. 2 Distribution of the SIDIS events vs. $Q^{2}$ (left) and vs. $y$ (right)
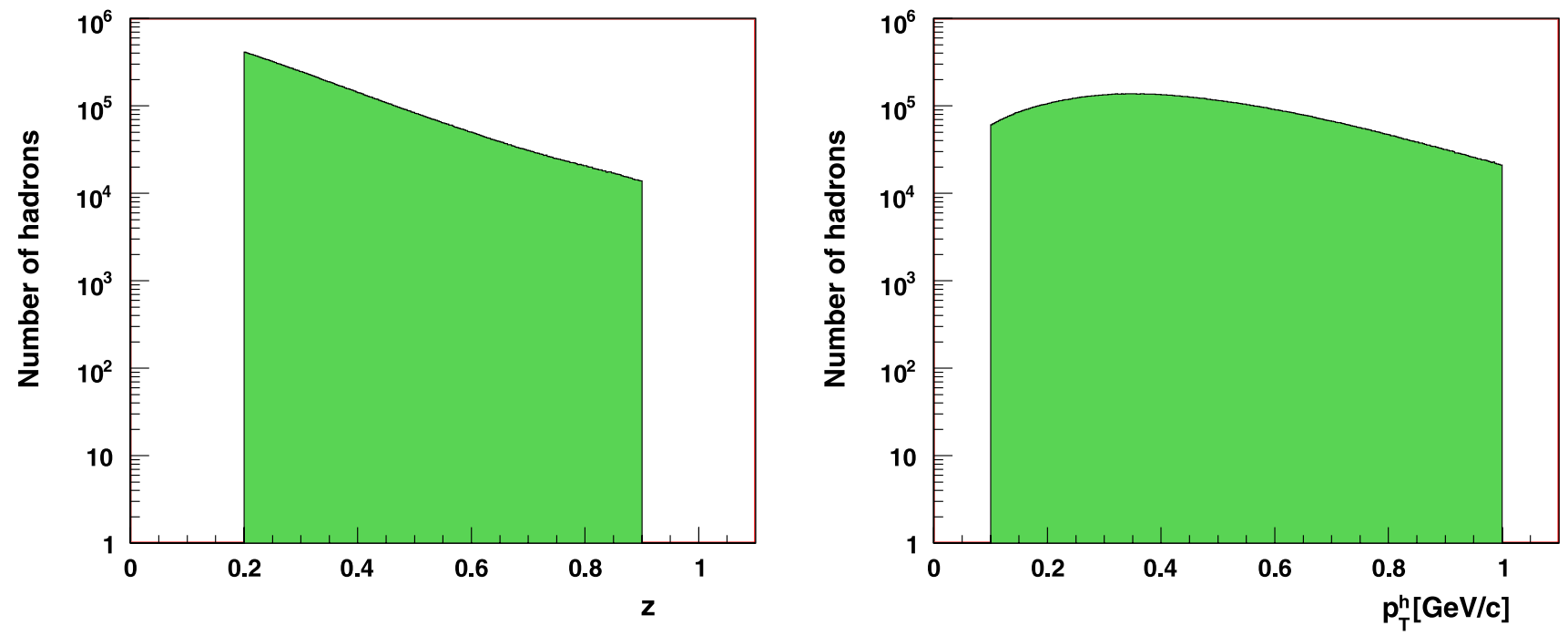

Fig. 3 Distribution of hadrons from the restricted kinematic region vs. $z$ (left $)$ and vs. $p_{T}^{h}$ (right)

have been calculated and fitted in $10 \phi$ bins. In a second step the dependence of $a^{f(\phi)}$ on each of the variables was studied while integrating over the other two variables. For this purpose fits of $a(\phi)$ have been performed in 10 bins of $\phi$ for each of the 6 bins in $x, z$, and $p_{T}^{h}$. In (9) we have disregarded the contribution of the $\cos 2 \phi$ term which could appear from $\mathrm{d} \sigma_{00}$ in the denominator of (4). This amplitude is expected [42-44] to not exceed $10 \%$ and would enter into (9) with the factor $a^{\text {const }}$ which is of order of $10^{-3}$ (see Table 1). This is beyond our accuracy. The same comments are valid for the contribution of the $\cos \phi$ and $\sin \phi$ amplitudes coming from the denominator of (4). However, their contributions are automatically taken into account by the corresponding fit parameters.
For the integrated sample the parameters characterising the $\phi$-modulation amplitudes are compatible with zero within 1-1.5 standard deviations. The $\phi$-independent parameters $a^{\text {const }}$ differ from zero and are almost equal for $h^{-}$ and $h^{+}$within the statistical errors. The fit parameters are given in Table 1. The correlation coefficients of the fit parameters do not exceed $15 \%$. Also given are the results for fits considering only a constant term, $a(\phi)=a^{\text {const }}$. The $\phi$-independent parts of the asymmetries appear due to the first term of $\mathrm{d} \sigma_{L L}$, which is proportional to the helicity PDF $g_{1 L} \equiv g_{1}$ convoluted with the PFF of unpolarised quarks in an unpolarised hadron (see (5)). For the isoscalar deuteron this contribution is expected to be only weakly dependent on the hadron charge, as is confirmed by the results. Moreover, 
these constants are related to the hadron SIDIS asymmetry $A_{1, d}^{h}(x)$ for deuterons (see below).

The dependence of the amplitudes of the $\phi$ modulation on the kinematic variables is shown in Figs. 4-8. The bin sizes are optimised to contain more than $10^{6}$ events in each bin. Some points are slightly shifted horizontally for clarity. Only statistical errors are shown. Systematic uncertainties are estimated to be much smaller then the statistical ones (see Sect. 6).

The asymmetry parameter $a^{\text {const }}(x)$ divided by the average muon polarisation and the virtual-photon depolarisation factor $D_{0} \approx\left|P_{\mu}\right| \sqrt{1-\epsilon^{2}}$ in the corresponding $x$ bin

Table 1 Best values of the $a(\phi)$ fit parameters for positive and negative hadrons from the five- and one-parameter fits

\begin{tabular}{lrrll}
\hline $\begin{array}{l}\text { Fit parameters } \\
\times 10^{4}\end{array}$ & \multicolumn{1}{l}{$h^{-}$} & \multicolumn{1}{l}{$h^{+}$} & $h^{+}$ \\
\hline$a^{\text {const }}$ & $23 \pm 17$ & $40 \pm 15$ & $23 \pm 16$ & $35 \pm 15$ \\
$a^{\sin \phi}$ & $15 \pm 23$ & $-30 \pm 21$ & - & - \\
$a^{\sin 2 \phi}$ & $30 \pm 23$ & $-24 \pm 21$ & - & - \\
$a^{\sin 3 \phi}$ & $40 \pm 24$ & $-10 \pm 21$ & - & - \\
$a^{\cos \phi}$ & $-4 \pm 24$ & $38 \pm 22$ & - & - \\
$\chi^{2} /$ n.d.f. & $6.1 / 5$ & $1.0 / 5$ & $10.4 / 9$ & \multicolumn{1}{l}{$7.0 / 9$} \\
\hline
\end{tabular}

is shown in Fig. 4. By definition, the value $a^{\text {const }}(x) / D_{0}$ is equal to the asymmetry $A_{1, d}^{h}(x)$ published earlier [15]. The agreement of these data with those of the present analysis demonstrates the internal consistency of the results obtained by different methods.

The $\sin \phi$ modulation amplitudes of the azimuthal asymmetry are shown in Fig. 5. Such a modulation is expected as a combined effect from the twist-3 PDFs $h_{L}$ and $f_{L}^{\perp}$ entering $\mathrm{d} \sigma_{0 L}$ as well as from the twist-2 transversity PDF $h_{1}$ and Sivers PDF $f_{1 T}^{\perp}$ entering $\mathrm{d} \sigma_{0 T}$ (see (5)), all contributing to the azimuthal asymmetries with a factor $M x / Q$. The individual PDF contributions can not be separated within a single experiment. The observed $x$ dependence of this amplitude is less pronounced in the COMPASS data than in the HERMES [38] data. ${ }^{3}$ The latter are obtained for leading pions, while our data include all hadrons and cover a much wider range in $x, Q^{2}$ and $W^{2}$. When restricting our kinematic region to that of HERMES for the amplitude $a^{\sin \phi}$ compatible results are obtained.

The amplitudes of the $\sin (2 \phi)$ modulation shown in Fig. 6 are small, consistent with zero within the errors. They

${ }^{3}$ The sign of HERMES data was inverted in order to match our definition of spin asymmetry by (4).
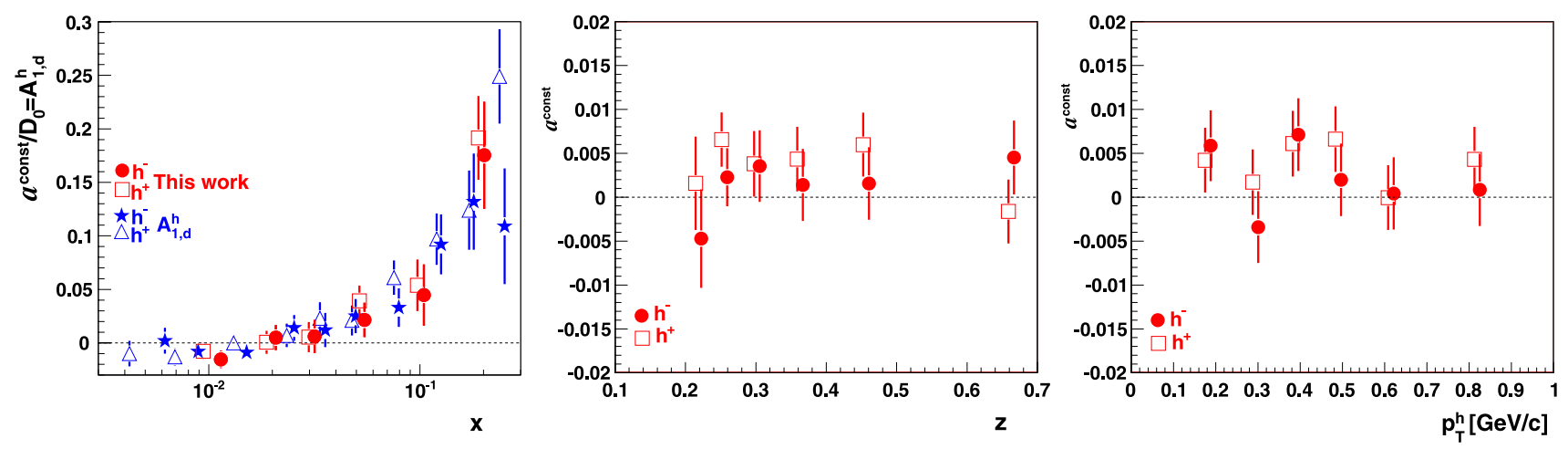

Fig. 4 Dependence of the $a^{\text {const }}$ parameter on the kinematic variables. The values of $A_{(1, d)}^{h}(x)$ from Ref. [15] are also shown
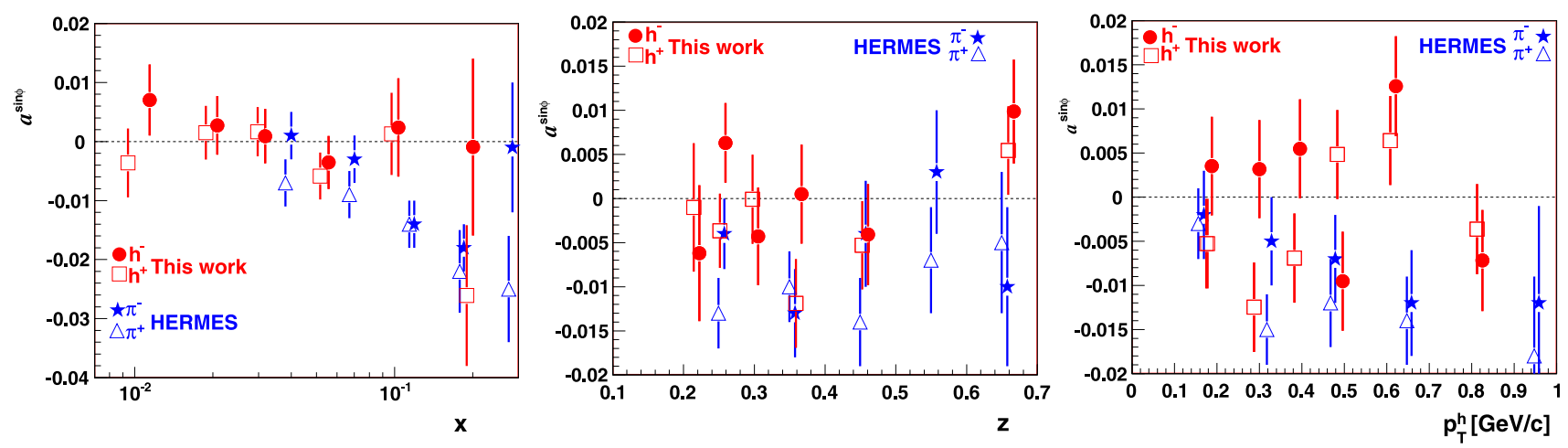

Fig. 5 Dependence of the modulation amplitude $a^{\sin \phi}$ on the kinematic variables and similar data of HERMES [38] for identified leading pions 

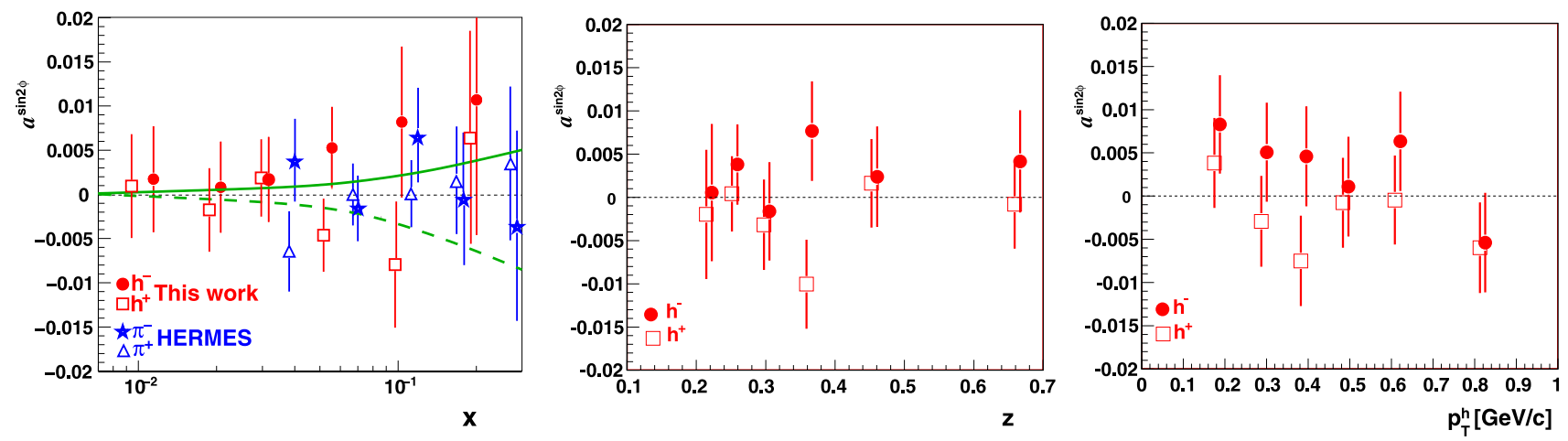

Fig. 6 Dependence of the modulation amplitude $a^{\sin 2 \phi}$ on the kinematic variables. The $x$ dependence of this amplitude is compared to the data and calculations by H. Avakian et al. [45] for HERMES kinematics ${ }^{3}$ for positive (solid line) and negative (dashed line) hadrons
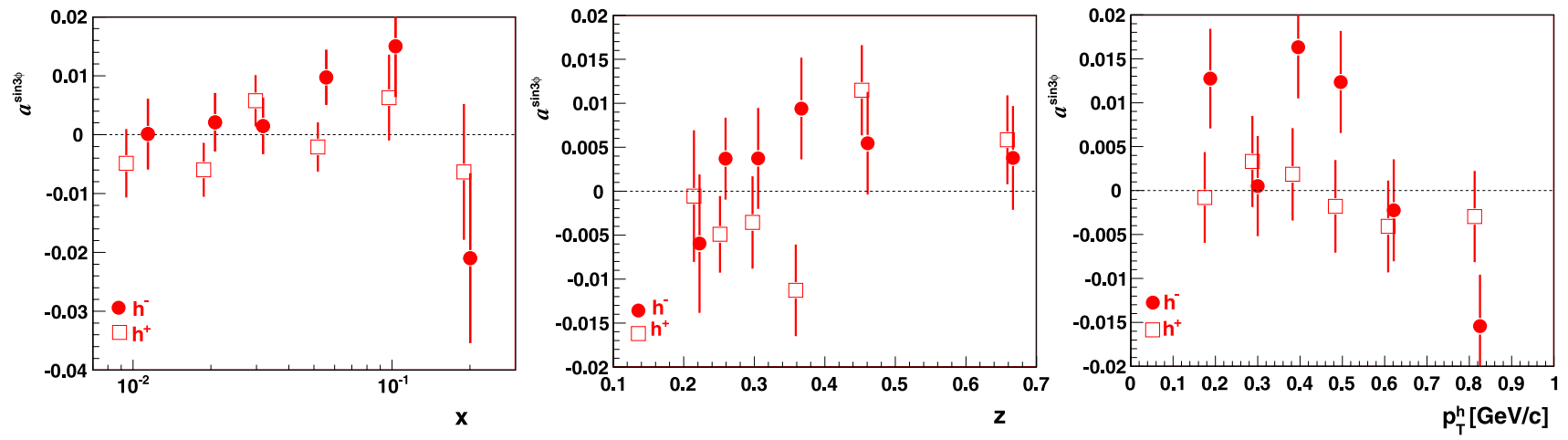

Fig. 7 Dependence of the modulation amplitude $a^{\sin 3 \phi}$ on the kinematic variables
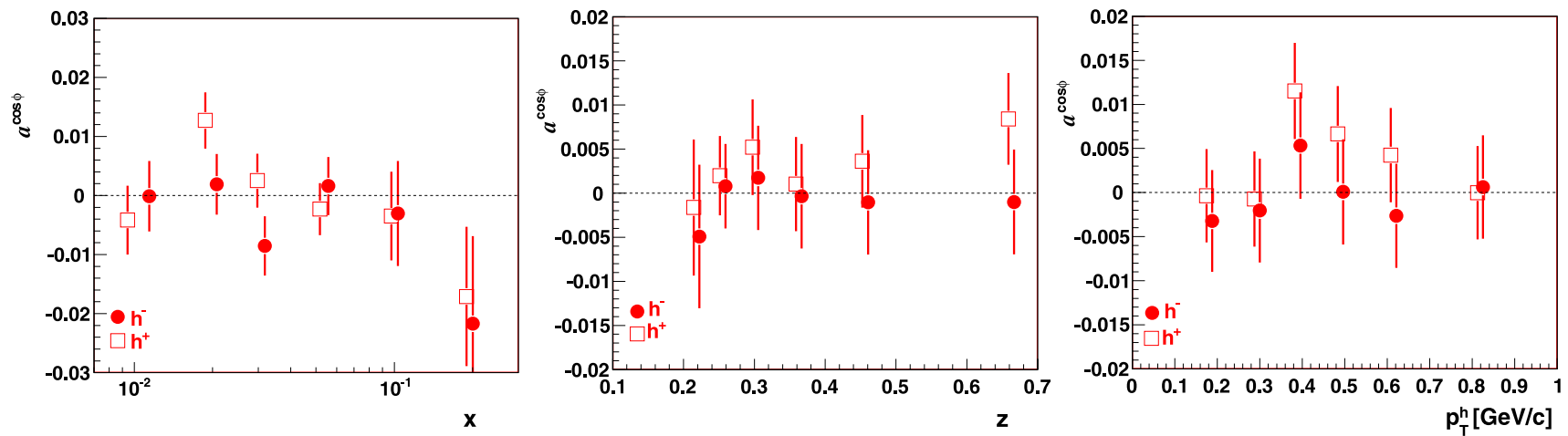

Fig. 8 Dependence of the modulation amplitude $a^{\cos \phi}$ on the kinematic variables

are due to the PDF $h_{1 L}^{\perp}$ in $\mathrm{d} \sigma_{0 L}$ (see (5)) which is approximately linked [45] to the transversity PDF $h_{1}$ by a relation of the Wandzura-Wilczek type.

The data on the modulation amplitude $a^{\sin 3 \phi}$ shown in Fig. 7 are compatible with zero as our results on the amplitude of the $\sin \left(3 \phi-\phi_{S}\right)$ modulation extracted from transversely polarised deuterons [40] $\left(\phi_{S}=0, \pi\right.$ for longitudinal target polarisation). This modulation would be due to the pretzelosity PDF $h_{1 T}^{\perp}$ in $\mathrm{d} \sigma_{0 T}$ and is suppressed by the factor $\tan \theta_{\gamma} \sim x M / Q$.
The $\cos \phi$ modulation of the azimuthal asymmetries for a longitudinally polarised target is studied here for the first time. The data presented in Fig. 8 are consistent with no variations of the modulation amplitudes vs. $x, z$ and $p_{T}^{h}$. This amplitude is proportional to the muon beam polarisation and would be due to a pure twist-3 PDF $g_{L}^{\perp}$ in $\mathrm{d} \sigma_{L L}$, an analogue to the Cahn effect [46, 47] in unpolarised SIDIS [42-44], and $g_{1 T}$ in $\mathrm{d} \sigma_{L T}$, suppressed by $\tan \theta_{\gamma} \sim x M / Q$. 

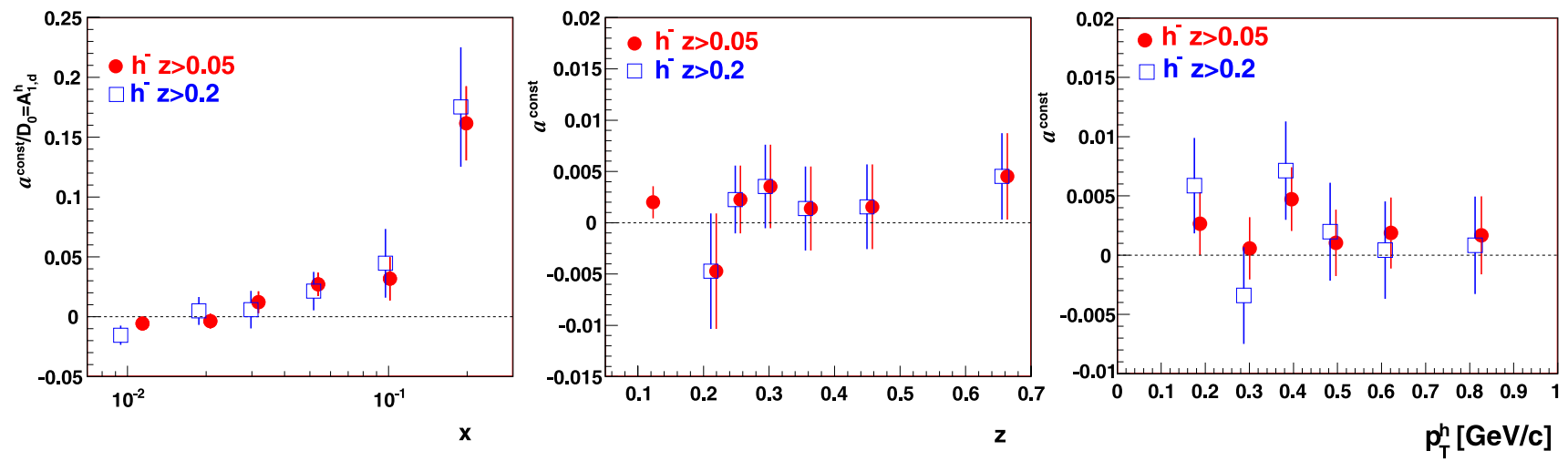

Fig. 9 Comparison of results for the $h^{-}$azimuthal asymmetry parameter $a^{\text {const }}(x) / D_{0}, a^{\text {const }}(z)$ and $a^{\text {const }}\left(p_{T}^{h}\right)$ for different regions: $z=0.05-0.9$ and $z=0.2-0.9$

\section{Stability of the results and systematics}

In the above analysis the $z$ cut $(z>0.2)$ has been applied to reject hadrons originating from the target fragmentation region. To check if a lower cut can affect the results presented in Table 1 and in Figs. 4-8, we have repeated the analysis with the cut $z>0.05$ and obtained consistent results. The results for the two different $z$ cuts are compared in Fig. 9 for the asymmetry parameters $a^{\text {const }}(x) / D_{0}, a^{\text {const }}(z)$, and $a^{\text {const }}\left(p_{T}^{h}\right)$. The $x$ dependence of this parameter does not depend on the applied $z$ cut, i.e. there is no influence of the target fragmentation region on the $x$ dependence for the selected sample of events down to $z=0.05$. The observed $p_{T}^{h}$ dependence looks smoother with higher statistics.

The compatibility of the final results on the azimuthal asymmetries obtained with the data taken in 2002-2004 has been checked by building distributions of "pulls"

$\frac{a_{i}-\langle a\rangle}{\sqrt{\sigma_{a_{i}}^{2}-\sigma_{\langle a\rangle}^{2}}}$,

where $a_{i}$ is the asymmetry in a given year for a given hadron charge and bin of the kinematic variables $x, z$, and $p_{T}^{h}$. The value $\langle a\rangle$ is the corresponding weighted mean over three years and $\sigma$ is the statistical error. The overall distribution of pulls for all $a_{i}$ measurements (540 entries, i.e. 5 asymmetries for positive and negative hadrons, 3 variables with 6 bins and 3 years of data taking) is shown in Fig. 10 together with its fit by a Gaussian. As expected, the distribution of pulls follows a standard distribution with mean close to zero and sigma equal to unity indicating that the fluctuations in the data are only statistical.

The double ratio (6) has been used to extract the asymmetries because of the cancellation of acceptance and flux. To check this cancellation, two different double ratios have

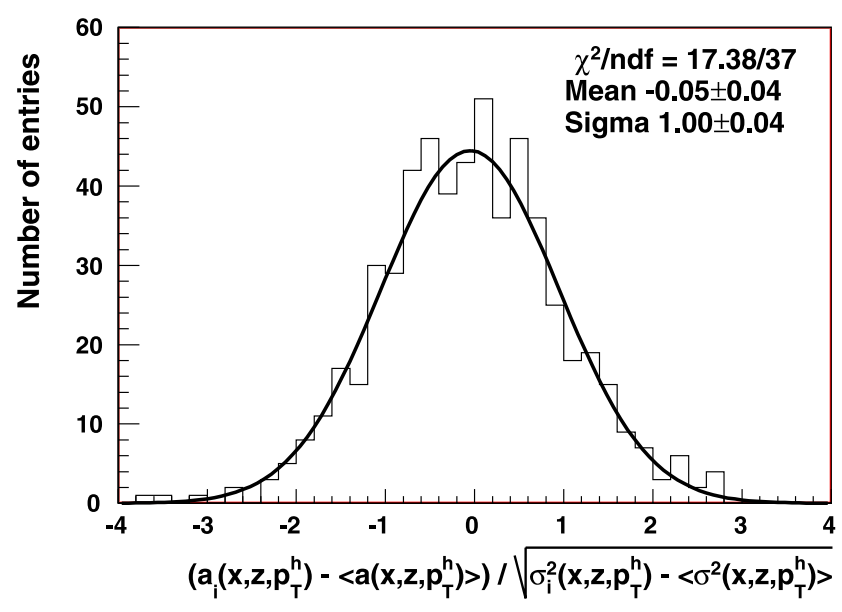

Fig. 10 Distribution of pulls for measurements of $a_{i}$

been constructed using the same number of events, namely

$F_{+}(\phi)=\frac{N_{++}^{U} N_{-+}^{D}}{N_{++}^{D} N_{-+}^{U}}$

and

$F_{-}(\phi)=\frac{N_{+-}^{U} N_{--}^{D}}{N_{+-}^{D} N_{--}^{U}}$

for the positive and negative solenoid field orientations, respectively. Substituting into (11), (12) the number of events by the corresponding expressions given in (7), one could see that these ratios depend neither on the acceptance nor on the DIS cross-section. The above equations should reflect the relative integrated muon fluxes squared for the measurements with different target polarisation but the same solenoid field orientation and they are a good check of acceptance cancellation in (6).

Indeed, the ratios (11), (12) for the selected events integrated over all kinematic variables are found to be independent of $\phi$ and the field orientations and are the same 


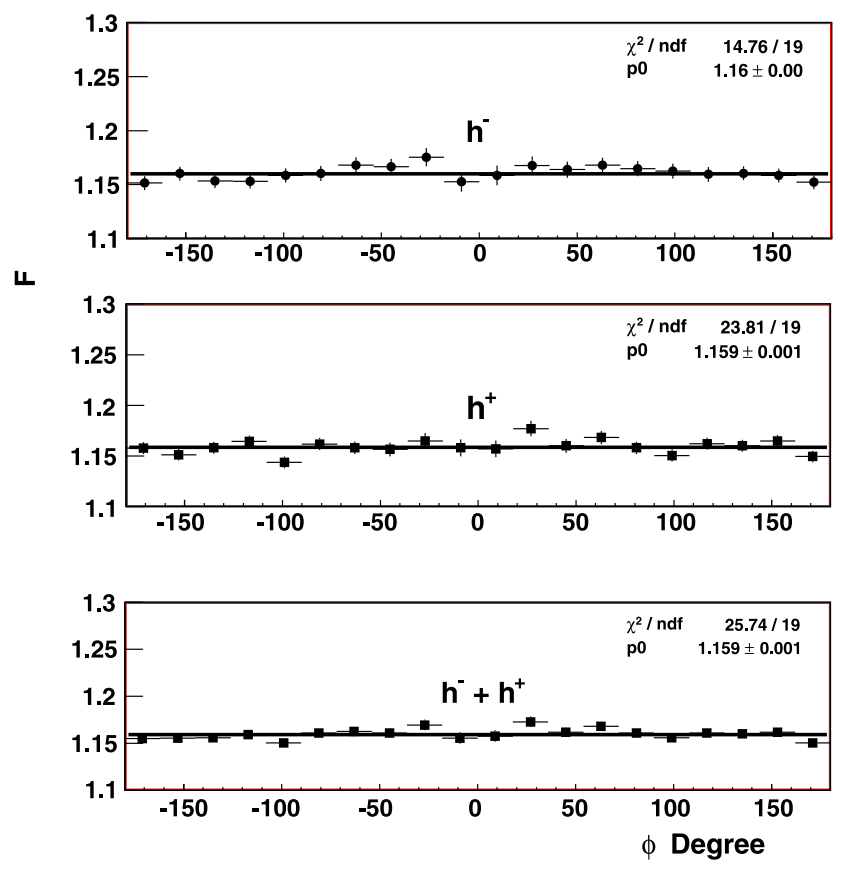

Fig. 11 Double ratios of event numbers for the weighted sum $F=F_{+}(\phi) \oplus F_{-}(\phi)$ demonstrating the $\phi$ stability of the data taking rates for negative (top), positive (middle) and all hadrons (bottom). The parameters $p_{0}$ represent the results of fits by a constant

for $h^{+}$and $h^{-}$(i.e., $\phi$-dependent acceptances $C_{f}^{t}(\phi)$ are really cancelled). This is illustrated in Fig. 11 where the weighted sums of $F=F_{+}(\phi) \oplus F_{-}(\phi)$ for negative and positive hadrons are shown together with results of their fit by a constant.

The compatibility and stability tests indicated no systematic effects. It is therefore assumed that the systematic uncertainties of the measured asymmetries due to variations of the acceptance are smaller than the statistical errors.

The uncertainties due to the target and beam polarisation are estimated to be of the order of $5 \%$ each. The uncertainty of the dilution factor, which takes into account the target material composition, is of the order of 2\% [11-14]. Each of them introduces the corresponding multiplicative uncertainty in the asymmetry measurement. When combined in quadratures, these errors give a global systematic multiplicative uncertainty of less than $7 \%$. The errors due to the additive radiative correction are negligible in the used kinematical region.

\section{Conclusions}

Azimuthal asymmetries $a(\phi)$ were studied in the production of positive and negative hadrons by $160 \mathrm{GeV}$ muons scattering off the longitudinally polarised deuterons. Integrated over the variables $x, z$, and $p_{T}^{h}$, all $\phi$-modulation amplitudes of $a(\phi)$ are consistent with zero, while the $\phi$-independent parts of the $a(\phi)$ distributions differ from zero and are equal for positive and negative hadrons within the statistical errors. In the study of $a(\phi)$ as a function of $x, z$, and $p_{T}^{h}$ the following results are obtained. The $\phi$-independent terms $a^{\text {const }}(x)$ of $a(\phi)$ are in agreement with our earlier results [15] and other published data on $A_{1, d}^{h}$, calculated by other methods and using different cuts. The amplitudes $a^{\sin \phi}$ are small and in general compatible with the HERMES data [38]. The amplitudes $a^{\sin 2 \phi}\left(x, z, p_{T}^{h}\right), a^{\sin 3 \phi}\left(x, z, p_{T}^{h}\right)$ and $a^{\cos \phi}\left(x, z, p_{T}^{h}\right)$ are consistent with zero within statistical errors.

These data will be useful to constrain models of the nucleon structure. The present parton model description of the SIDIS cross-sections involves a considerable number of PDFs depending on the longitudinal and transverse components of the nucleon spin. We believe that our data will help to assess which PDFs are important in the description of the nucleon structure.

Acknowledgements We gratefully acknowledge the support of the CERN management and staff and the skill and effort of the technicians of our collaborating institutes. Special thanks go to V. Anosov and V. Pesaro for their technical support during the installation and running of this experiment.

Open Access This article is distributed under the terms of the Creative Commons Attribution Noncommercial License which permits any noncommercial use, distribution, and reproduction in any medium, provided the original author(s) and source are credited.

\section{References}

1. M.J. Alguard et al. (SLAC Collaborations), Phys. Rev. Lett. 37, 1261 (1976)

2. G. Baum et al. (SLAC Collaborations), Phys. Rev. Lett. 51, 1135 (1983)

3. J. Ashman et al. (European Muon Collaboration), Phys. Lett. B 206, 364 (1988)

4. J. Ashman et al. (European Muon Collaboration), Nucl. Phys. B 328, 1 (1989)

5. B. Adeva et al. (Spin Muon Collaboration), Phys. Rev. D 58, 112001 (1998)

6. A. Airapetian et al. (HERMES Collaboration), Phys. Rev. D 71, 012003 (2005). arXiv:hep-ex/0407032

7. A. Airapetian et al. (HERMES Collaboration), Phys. Rev. D 75, 012007 (2007). arXiv:hep-ex/0609039

8. J. Yun et al. (CLAS Collaboration), Phys. Rev. C 67, 055204 (2003). arXiv:hep-ex/0212044

9. R. Fatemi et al. (CLAS Collaboration), Phys. Rev. Lett. 91, 222002 (2003). arXiv:nucl-ex/0306019

10. K.V. Dharmawardane et al. (CLAS Collaboration), Phys. Lett. B 641, 11 (2006). arXiv:nucl-ex/0605028

11. E.S. Ageev et al. (COMPASS Collaboration), Phys. Lett. B 612, 154 (2005). arXiv:hep-ex/0501073

12. V.Y. Alexakhin et al. (COMPASS Collaboration), Phys. Lett. B 647, 8 (2007). arXiv:hep-ex/0609038

13. M. Alekseev et al. (COMPASS Collaboration), Phys. Lett. B 680, 217 (2009). arXiv:0905.2828 [hep-ex]

14. M.G. Alekseev et al. (COMPASS Collaboration), Phys. Lett. B 690, 466 (2010). arXiv: 1001.4654 [hep-ex] 
15. M. Alekseev et al. (COMPASS Collaboration), Phys. Lett. B 660, 458 (2008). arXiv:0707.4077 [hep-ex]

16. D.W. Sivers, Phys. Rev. D 41, 83 (1990)

17. J.C. Collins, Nucl. Phys. B 396, 161 (1993). arXiv:hep-ph/ 9208213

18. A. Kotzinian, Nucl. Phys. B 441, 234 (1995). arXiv:hep-ph/ 9412283

19. A.M. Kotzinian, P.J. Mulders, Phys. Rev. D 54, 1229 (1996). arXiv:hep-ph/9511420

20. P.J. Mulders, R.D. Tangerman, Nucl. Phys. B 461, 197 (1996) [Erratum ibid. B 484, 538 (1997)]

21. D. Boer, P.J. Mulders, Phys. Rev. D 57, 5780 (1998)

22. H. Avakian, A.V. Efremov, P. Schweitzer, F. Yuan, Phys. Rev. D 81, 074035 (2010). arXiv:1001.5467 [hep-ph]

23. M. Anselmino, M. Boglione, U. D’Alesio, S. Melis, F. Murgia, A. Prokudin, Phys. Rev. D 81, 034007 (2010). arXiv:0911.1744 [hep-ph]

24. A. Bacchetta et al., J. High Energy Phys. 0702, 093 (2007). arXiv:hep-ph/0611265

25. A. Bravar (Spin Muon Collaboration), Nucl. Phys. Proc. Suppl. 79, 520 (1999)

26. A. Airapetian et al. (HERMES Collaboration), Phys. Rev. Lett. 84, 4047 (2000). arXiv:hep-ex/9910062

27. A. Airapetian et al. (HERMES Collaboration), Phys. Rev. D 64, 097101 (2001). arXiv:hep-ex/0104005

28. H. Avakian et al. (CLAS Collaboration), Phys. Rev. D 69, 112004 (2004). arXiv:hep-ex/0301005

29. H. Avakian, L. Elouadrhiri (CLAS Collaboration), Phys. Part. Nucl. 35, S114 (2004)

30. A. Airapetian et al. (HERMES Collaboration), Phys. Rev. Lett. 94, 012002 (2005). arXiv:hep-ex/0408013

31. A. Airapetian et al. (HERMES Collaboration), Phys. Rev. Lett. 103, 152002 (2009). arXiv:0906.3918 [hep-ex]
32. A. Airapetian et al. (HERMES Collaboration), Phys. Lett. B 693, 11 (2010). arXiv:1006.4221 [hep-ex]

33. V.Y. Alexakhin et al. (COMPASS Collaboration), Phys. Rev. Lett. 94, 202002 (2005). arXiv:hep-ex/0503002

34. E.S. Ageev et al. (COMPASS Collaboration), Nucl. Phys. B $\mathbf{7 6 5}$, 31 (2007). arXiv:hep-ex/0610068

35. M. Alekseev et al. (COMPASS Collaboration), Phys. Lett. B 673, 127 (2009). arXiv:0802.2160 [hep-ex]

36. M. Alekseev et al. (COMPASS Collaboration), Phys. Lett. B 692 , 240 (2010). arXiv:1005.5609 [hep-ex]

37. A. Airapetian et al. (HERMES Collaboration), Phys. Lett. B 622, 14 (2005). arXiv:hep-ex/0505042

38. A. Airapetian et al. (HERMES Collaboration), Phys. Lett. B 562, 182 (2003). arXiv:hep-ex/0212039

39. H. Avakian et al. (CLAS Collaboration), arXiv:1003.4549 [hepex]

40. A. Kotzinian (COMPASS Collaboration), in Munich 2007, Deepinelastic scattering, pp. 647-650. arXiv:0705.2402 [hep-ex]

41. P. Abbon et al. (COMPASS Collaboration), Nucl. Instrum. Methods A 577, 455 (2007). arXiv:hep-ex/0703049

42. M. Arneodo et al. (European Muon Collaboration), Z. Phys. C 34, 277 (1987)

43. F. Giordano, R. Lamb (On behalf of the HERMES Collaboration), AIP Conf. Proc. 1149, 423 (2009). arXiv:0901.2438 [hep-ex]

44. A. Bressan (COMPASS Collaboration), arXiv:0907.5511 [hepex]

45. H. Avakian et al., Phys. Rev. D 77, 014023 (2008). arXiv:0709. 3253 [hep-ph]

46. R.N. Cahn, Phys. Lett. B 78, 269 (1978)

47. R.N. Cahn, Phys. Rev. D 40, 3107 (1989) 